\title{
Application of the full-waveform inversion techniques to the estimation of the sound velocity structure in the ocean
}

\author{
Yukihiro KIDA $^{1}$, Hitoshi MIKADA ${ }^{1}$, Tada-nori GOTO ${ }^{1,}$ Junichi TAKEKAWA $^{1}$ \\ ${ }^{1}$ Geophysics Lab., Dept. of Civil and Earth Res. Eng., Kyoto University
}

This study investigates the effectiveness and the applicability of the full waveform inversion (FWI) method to estimate underwater sound velocity structures. We use the frequency domain full waveform inversion method in this study. In this study, the FWI is applied to the shallow-acoustic tomography first, and then we show some prospects of application to the long-range ocean acoustic tomography. We used an optimal 9 point finite difference frequency domain method for shallow acoustic tomography and the wide angled parabolic equation method for long-range acoustic tomography. We use an adjoint-state method for the calculation of the gradient in an iterative inversion based on a pre-conditioned conjugate gradient method. We first demonstrate results from a FWI method applied to a VCS experiment field data in Lake Biwa. In spite of very limited path condition using only direct arrival wave, the full waveform inversion method could describe the horizontal velocity structure possibly due to seasonal thermocline in the lake. Then, we applied the FWI method to the synthetic dataset of long-range acoustic propagation. We conclude that the FWI method could be the key success factor for the higher resolution at estimation of underwater sound velocity structure.

\section{INTRODUCTION}

Ocean Acoustic Tomography (OAT) is one of the methods to estimate the velocity structure of ocean using acoustic wave propagation and some elements related to the sound velocity that is a function of temperature, salinity, fluid velocity, etc. OAT was proposed by Munk et al. (1995) as a remote sensing technique for large scale ocean monitoring using low frequency acoustic waves. The concept of OAT is based on a ray-theoretical way using the travel-time inversion (TTI) techniques that aim to minimize the difference of arrival times between the observed and the calculated through a ray-theoretic modeling along eigenrays connecting sources and receivers. Instead of the ray-paths, some studies use the travel time sensitivity kernels that derived from the wave equation by the first order Born approximations. TTI techniques are relatively robust and provide images with a reasonable computational time. However the resolution of TTI method is limited within the scale of the Fresnel volume that is closely connected to travel time sensitivity kernels. Furthermore, it cannot utilize the additional information involved in reflected, refracted or guided waves because all information is integrated into the calculated ray-paths. These limits make it difficult to obtain the small scaled or minute information of the velocity structure.

In seismic tomography, the demands for the high-resolution have developed the waveform inversion methods. Full waveform inversion (FWI) method is one of the representative methods of wave form inversion methods that use the waveform as the cost function of the inversion. Generally, full wave field modeling methods based on the wave equations are used for forward modeling in FWI, so all structures that affect the recorded waveforms such as the velocity, attenuation, bulk modulus, density or etc. are considered for the inversion process. However, there are quite a few cases of waveform inversion techniques to the estimation of underwater acoustic velocity structure and it is considered to be important to take waveform inversion approach for higher resolution in tomography results. Some characteristics of the underwater acoustic environment are different from the seismic environment; low contrast of the velocity fields, weak attenuation, no sear waves, use of controlled wave sources and etc. Therefore it is important validate the applicability of waveform inversion approach to OAT.

This study focuses on the investigation of the applicability of the FWI method to the estimation of underwater acoustic velocity structure. In this study, after a short introduction of the 
inversion method, we present the application of FWI to the synthetic data and examine the efficiency of the method by comparing the result from TTI. Then we show the application example to the field experiment data with vertical cable seismic (VCS) system at Lake Biwa in Japan. Then we present the application result of synthetic data from long-range acoustic model that data contains the multi-pass waves of direct wave, reflected wave from surface or bottom and the channel wave passing through SOFAR channel. The results show high applicability of the method and prove that the FWI method is effective method to the estimation of underwater acoustic structure with high resolution.

\section{METHOD}

\section{A) Forward modeling methods (short-range)}

The frequency-domain wave equation is described as eq.1. In the frequency domain, the wave equation is described as a system of the linear equation.

$$
\mathbf{A}(\mathbf{x}, \omega) \mathbf{p}(\mathbf{x}, \omega)=-\mathbf{s}(\mathbf{x}, \omega)
$$

Where, $\mathbf{A}$ is the impedance matrix, $\mathbf{p}$ is the pressure wave field vector, $\mathbf{s}$ is the wave source and $\omega$ is the angular frequency. $\mathbf{A}$ is a function of the angular frequency and the velocity field. To solve the inverse of huge impedance matrix $\mathbf{A}$ is not practical or nearly impossible. In the short range propagation, we use the 2-D acoustic finite difference frequency domain (FDFD) method for the frequency domain forward modeling with 9-point optimal method to build a sparse and complex valued matrix A. To solve multi-source problem, an LU decomposition scheme is used to solve linear system in eq.2. The matrix $\mathbf{A}$ is independent from the sources term $\mathbf{s}$, therefore, once the matrix has been decomposed, it can be used to solve the pressure distribution for the other additional sources with minimal computational costs.

The computational cost of long-range acoustic modeling is one of the most difficult problems in the computational ocean acoustics. The long-range acoustic propagation in the ocean is approximated as the cylindrical diffusion. We use the wide angled parabolic equation (PE) method for the forward modeling of long-range case. The frequency domain acoustic wave equation in cylindrical coordinates is described as follows.

$$
\frac{\partial^{2} p}{\partial r^{2}}+\frac{1}{r} \frac{\partial p}{\partial r}+\frac{\partial^{2} p}{\partial z^{2}}+k_{0}^{2} n^{2} p=-S
$$

Where $\mathrm{k}$ is the wave number and $\mathrm{n}$ is the index of the refraction. In PE method, forward going wave of the acoustic Helmholtz equation in cylindrical coordinates is solved by Pade approximation (Jensen et al., 2011).

\section{B) Full Waveform Inversion method}

Full waveform inversion (FWI) method is based on the minimizing a cost function that measures the difference between the calculated and acquired data. We defined the L2 norm of the data residual vector for the misfit and objective function in the least square problem in FWI (eq.3 and 4).

$$
\begin{gathered}
E(\mathbf{m})=\delta \mathbf{p}^{*} \delta \mathbf{p} \\
\delta \mathbf{p}=\mathbf{p}_{o b s}-\mathbf{p}_{c a l}
\end{gathered}
$$

Where $\mathrm{E}$ is the cost function, $\mathbf{m}$ is the model parameters, and $\delta \mathbf{p}$ is the data residual vector calculated by the differences at the receiver positions between observed wavefield $p_{o b s}$ and the modeled wavefield $p_{c a l}$ for each pair of source and receiver. * denotes the adjoint (transpose conjugate) operator. The value $\mathrm{E}$ is minimized iteratively by calculating the gradient or the steepest descent direction at iteration $\mathrm{k}$ with the adjoint-state method.

In frequency-domain FWI, the gradient is acquired by calculating the cross-spectrum of the complex spectrum of the pressure wave-field as shown in eq.5;

$$
\begin{aligned}
\gamma(\mathbf{x})= & \frac{1}{\mathbf{V}(\mathbf{x})^{3}} \sum_{s} \sum_{r} \int \omega^{2} P_{c a l}\left(\mathbf{x}, \omega ; \mathbf{x}_{s}\right) \\
& \times \delta P^{*}{ }_{b a c k}\left(\mathbf{x}, \omega ; \mathbf{x}_{s}\right) d \omega
\end{aligned}
$$

where $\mathrm{P}$ is the complex spectrum of the pressure wave-field, $\gamma$ is the gradient of the misfit functio, "back" indicates the reverse time propagation using the residual pressure wave-field $\delta \mathrm{P}$ shown in eq. 4 as the source wave from the receiver location. The small letters $\mathrm{s}$ and $\mathrm{r}$ under the summation in eq.5 indicate sources and receivers respectively.

The phase of the calculated wave field is crucial for the stability of the inversion because the gradient is obtained by the cross-spectrum of the complex pressure field as eq.5. 'cycle skipping' or 'phase rapping' that result from the $2 \pi$ uncertainty of in determining the phase is a basic problem of the FWI. So the frequency of the waveforms or starting model of the inversion should be chosen carefully. The inversion process is started with low frequency component and gradually changes the frequency band as including the higher frequency part.

To speed up convergence, a preconditioned conjugate gradient method can be used as follows:

$$
\boldsymbol{\lambda}_{k}=-\left(\operatorname{diag}\left(\mathbf{H}_{a}\right)-\boldsymbol{\kappa} \mathbf{I}\right)^{-1} \boldsymbol{\gamma}_{k}
$$




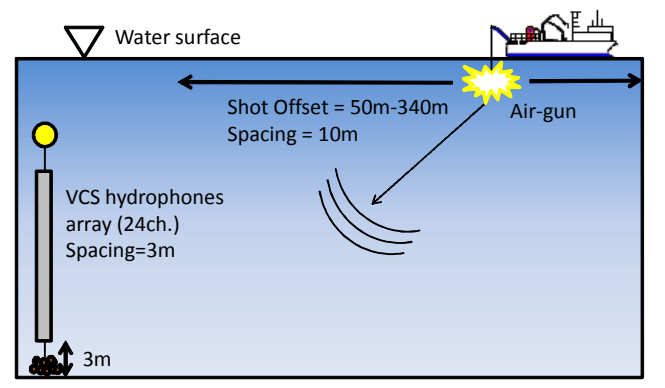

Fig.5 Acquisition geometry of the field experiment

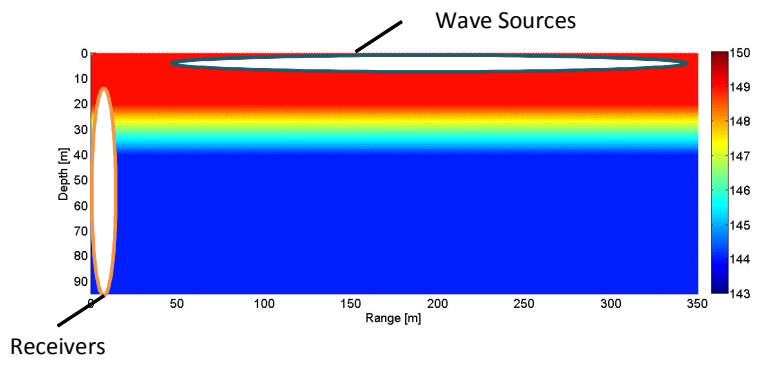

Fig.6 Initial model for the inversion

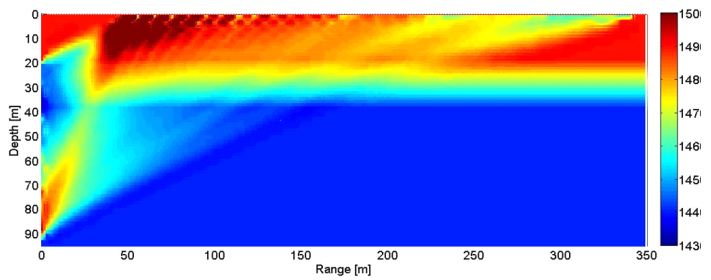

Fig.7 Inversion result of TTI

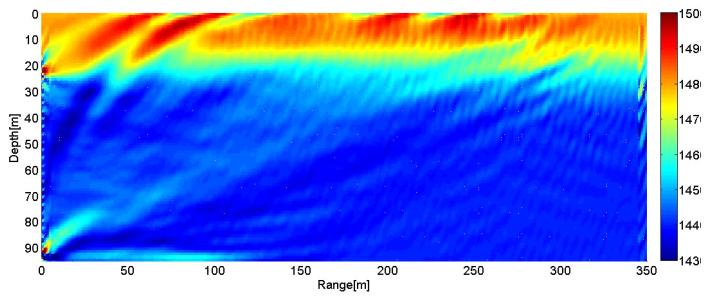

Fig.8 Inversion result of FWI Velocity profile with depth

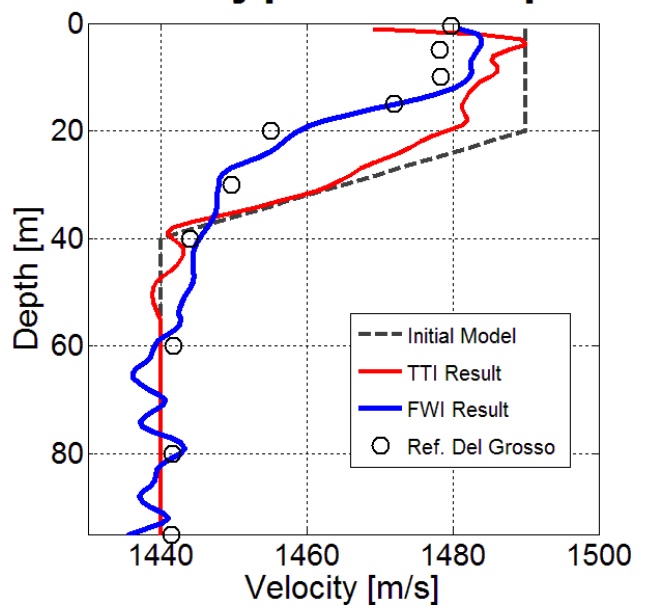

Fig.9 Vertical Profile at $125 \mathrm{~m}$ offset

$$
\mathbf{H}_{a}=\mathbf{J}^{T} \mathbf{J}
$$

where the preconditioning term $H_{a}$ is the approximate Hessian of the misfit function E, I is the identity matrix and $\kappa$ is the dumping factor to avoid singular values. The approximate Hessian matrix is calculated by the products of Jacobian matrix shown in eq.7. The preconditioned conjugate gradient is then calculated as followings:

$$
\boldsymbol{\delta}_{k}=\boldsymbol{\lambda}_{k}+\beta_{k} \boldsymbol{\delta}_{k-1}
$$

Where $\beta$ is the conjugate scaling factor by the Polak-Ribiere's method shown in eq.8.

$$
\beta_{k}=\frac{\left\langle\lambda_{k}, \lambda_{k}-\lambda_{k-1}\right\rangle}{\left\langle\lambda_{k-1}^{*}, \lambda_{k-1}\right\rangle}
$$

The model is then updated using following equation:

$$
\mathbf{m}_{k+1}=\mathbf{m}_{k}-\alpha_{k} \Delta \boldsymbol{\lambda}_{k}
$$

where $\alpha$ is the optimal positive scalar step length from a line search along the gradient direction using a parabolic fit.

\section{APPLICATION TO FIELD DATA IN SHORT RANGE TOMOGRAPHY}

The VCS acoustic experiment data in Lake Biwa in November 2009 was used in this study. Fig.5 shows the acquisition geometry of the VCS experiment. The research field is selected around the deepest point of Lake Biwa where the depth of water layer is more than $90 \mathrm{~m}$. The air-gun is used as a wave source and the receiver consists of 24 hydrophones array with $3 \mathrm{~m}$ vertically spacing above the bottom of the lake. The air-gun is towed by ship horizontally along the survey line and is shot every $10 \mathrm{~m}$. We use 30 shot records which offsets are between about $60 \mathrm{~m}$ and $350 \mathrm{~m}$ for the inversion. The inversion process is started with $62 \mathrm{~Hz}$ signals and gradually changed up to including $240 \mathrm{~Hz}$. We use only the first-arrival direct wave for the inversion because we don't have the bathymetry information of the survey area.

Fig. 6 shows the initial model for the inversion. We set a horizontal velocity structure having transition zone reflected the thermocline around $30 \mathrm{~m}$ depth. Fig. 7 and fig. 8 show the results of TTI and FWI respectively. Fig.9 shows the vertical profile of the velocity structure in $125 \mathrm{~m}$ offset. The solid red line is the result of TTI, solid blue line is the result of FWI, the black dashed line is the initial model and opened circle shows the field truth calculated from the temperature measurement data by empirical formula. The TTI result is averaged along the ray-path and couldn't revise the boundary of the thermocline. The FWI method could describe the boundary of the thermocline much in much higher resolution and the velocity distribution is very well matched to the measurement data. 


\section{APPLICATION TO LONG-RANGE ACCOUSTIC DATASET}

In long-range model, we assumed the observation of the velocity changes in the neighborhood of the SOFAR channel. Fig. 10 shows the velocity model that has two velocity anomalies near the center of SOFAR channel and the calculation area surrounded by the black dashed rectangle. The wave-source and receivers array are placed on the left and right side of model respectively. The water column is $50 \mathrm{~km}$ width and $3 \mathrm{~km}$ depth and the $3 \mathrm{~dB} /$ wavelength absorbing sediment layer is set under the depth of $3 \mathrm{~km}$. We use the Gaussian beam source of $100 \mathrm{~Hz} \pm 10 \mathrm{~Hz}$ as the wave source. The beam is emitted in 5 degree of lower parts and the radiation angle is \pm 5 degree. The length of the receivers array is $400 \mathrm{~m}$ and is placed in the center of SOFAR channel. The receivers are spaced each $5 \mathrm{~m}$. We used the frequency component of the 90,100 and $110 \mathrm{~Hz}$ for the inversion.

Fig.11 shows the result of FWI. The velocity structures are imaged in the near position of the velocity anomalies in fig.10. Fig.12 and Fig.13 shows the time-domain observed waveforms and residual waveforms in the receiver array. The residual waveforms are converged to well not only the strong direct channel guided waves but also the multiple waves recorded after the direct waves.

\section{CONCLUSION}

The performance of the FWI to underwater acoustic data in the estimation of acoustic phase velocities was examined for both long-range synthetic data and VCS (Vertical Cable Seismic) field experiment data. The VCS result provides the resolved velocity anomalies with much higher resolution than TTI method. Long-range result indicates the FWI method could be applied in multi-pass guided wave environment. These results in this study showed the high efficiency of FWI for underwater sound velocity estimation.

\section{REFERENCES}

1) Munk, W., Worcester, P., and Wunsch, C. ( 1995 ) : Ocean Acoustic Tomography, Cambridge Univ. Press

2) Tarantola, A. (1984): Inversion of seismic reflection data in the acoustic approximation: Geophysics, 49, (8)

3) Jo. C., Shin, C., Suh, J. (1996) : An optimal 9-point, finite-difference, frequency-space $2 \mathrm{~d}$ scalar wave extrapolator: Geophysics, 61, (2)

4) Virieux, J., Operto, S., 2009, An overview of

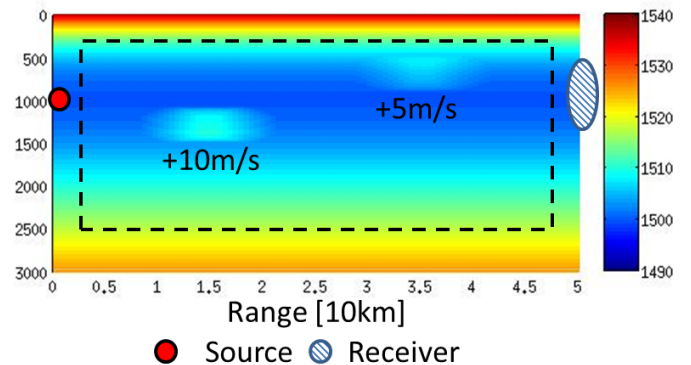

Fig.10 Upper: The velocity model of the long-range propagation. Two smoothed velocity anomalies are in the horizontal velocity model by Munk's profile.

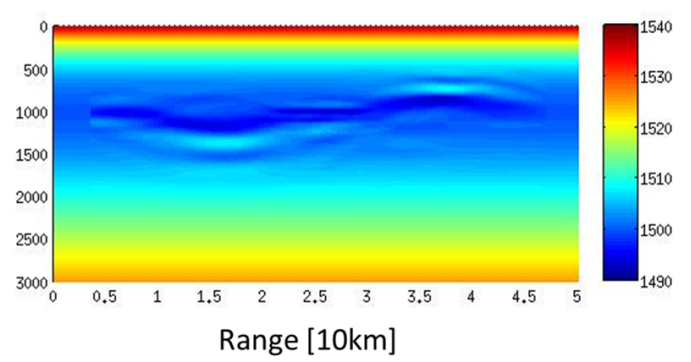

Fig.11 The inversion result. The horizontal velocity model by Munk's profile is used for the initial model.

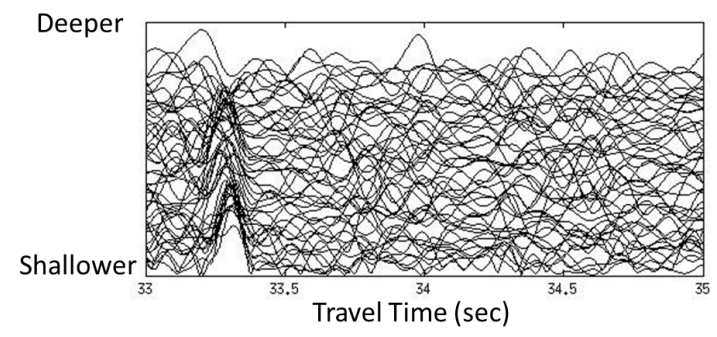

Fig.12 Observed Waveforms in time-domain synthetics by FFT.

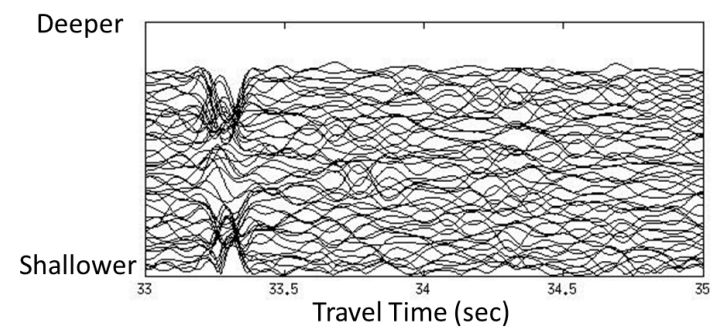

Fig.13 Residual waveforms of inversion result.

full-waveform inversion in exploration geophysics: Geophysics, 74, (6)

5) Jensen, F.B., Kuperman, W.A., Porter, M.B., Schmidt, H. (2011) : Computational Ocean Acoustics second edition, Springer 